\title{
Monitoring Choreographed Services
}

\author{
L. Ardissono and R. Furnari and A. Goy and G. Petrone and M. Segnan \\ Dipartimento di Informatica, Università di Torino \\ Corso Svizzera 185, 10149 Torino, Italy
}

\begin{abstract}
Web Service choreography management challenges the research on Service Oriented Architectures: different from Web Service composition, where a central orchestrator process invokes the Web Service suppliers, a choreographed service is decentralized and it is based on the coordination of a set of Web Service suppliers having a partial view of the overall service. In this paper, we present a framework which supports the monitoring of the progress of a choreographed service, the early detection of faults and the notification of the Web Services affected by the faults.
\end{abstract}

\section{INTRODUCTION}

In the research on Service Oriented Architectures [9], the management of supply chains has been based on processes which orchestrate the execution of services within possibly complex workflows. For instance, the WS-BPEL language [7] is used to specify parameters and execution order of the invocations of operations on Web Service suppliers, as well as of the internal activities to be performed by the composite Web Service. Moreover, Abstract Processes, based on the WS-BPEL syntax, describe the invocation protocol of a conversationally complex Web Service supplier; this specification enables consumers to invoke the operations without violating the suppliers' business logics.

Web Service orchestration has been introduced to manage hierarchical supply chains in which a centralized entity invokes the suppliers. However, it has been recognized that, even in Enterprise Application Integration scenarios, the management of a non trivial supply chain often imposes the decentralized management of the composite service [5], e.g., in order to deal with the business requirements of suppliers which serve multiple consumers. In such situations, the cooperating Web Services have partial views of the overall service and they have to coordinate with each other in a complex multi-party conversation denoted as choreography [10]. The successful execution of the choreographed service thus depends on at least two factors: when the service is designed, Web Service suppliers have to be bound to each other (possibly by employing mediation services for interoperability purposes [6]); at service execution time, they have to perform operations successfully and in due time.

In this paper we propose a framework which supports the monitoring of the progress of a choreographed service, the early detection of faults and the notification of the Web Services affected by the faults. In our framework, a Monitor Web Service tracks the execution of the cooperating Web Services by analyzing their conversational behavior. During the execution of the choreographed service, the Monitor is informed about the messages sent or received by the cooperating Web Services and about their execution state. The Monitor utilizes this information to check whether the overall service correctly progresses, i.e., if the message flow among the Web Services is consistent with the choreography. If any fault occurs, the Monitor evaluates whether the choreographed service can still complete and informs the Web Services, in order to let them react to the occurred problem. For generality purposes, we assume that the Monitor does not have any information about the internal implementation of the Web Services. Thus, our framework relies on the analysis of messages.

Our choreography framework builds on WSCoordination [2] to manage the coordination context between the Web Services but it replaces the Web Services Coordinator with a Web Services Monitor which proactively checks the progress of the choreographed service and propagates the coordination information. In the rest of this paper, Section 2 shows the benefits of monitoring on a sample scenario, Section 3 presents our framework, and Section 4 discusses some related work and concludes the paper.

\section{MotiVATING EXAMPLE}

Monitoring the progress of a choreographed service and notifying the cooperating Web Services about execution problems enables to avoid delays in execution and to react to faults in timely fashion. In fact, the data dependency chains among different Web Services may be long and a Web Service might become aware that it is affected by an execution problem occurred in another Web Service after several messages have been sent. We show this point on a simple example from a Business to Consumer (B2C) e-commerce scenario.

In a typical B2C service, the online store is the contact point with the end customer; given the customer's orders, the store invokes a warehouse to prepare the requested parcel. In turn, the warehouse service might inquire with some shippers about the cost of sending the parcel to the 


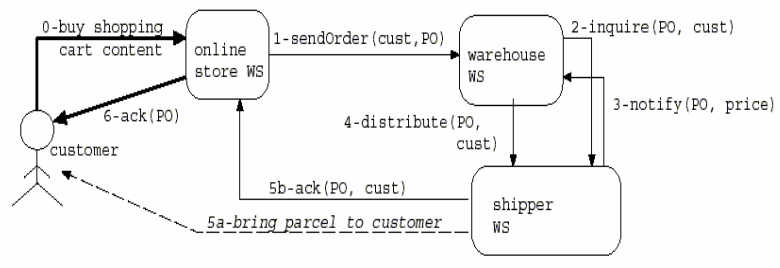

Fig. 1. Collaboration Diagram of a choreographed service.

customer in order to choose the most convenient one. Thus, the online store would not directly control the shipper's activities. However, in order to be able to inform the end customer about possible delivery problems (e.g., delays), the online store needs an acknowledgement from the selected shipper service as soon as the parcels have been sent out. Figure 1 shows the messages exchanged by the cooperating Web Services in the described scenario.
Now, suppose that, after having received an order from the warehouse, the selected shipper service becomes unavailable because of a software problem. Then, the online store would wait for the shipper's acknowledgment until a possibly long time out expires, and it would not be able to inform the end customer about the delay in a timely fashion. By monitoring the situation, the time out might be anticipated and the online store might be informed about the problem as soon as possible.

This example is deliberately simple but it should be noticed that, in a realistic scenario, the number of cooperating Web Services might be large and the dependency graph very complex. Thus, several service consumers might be blocked by the failure of a supplier. Having a global view of the situation is therefore strategic to notify the services which cannot continue their own execution in due time.

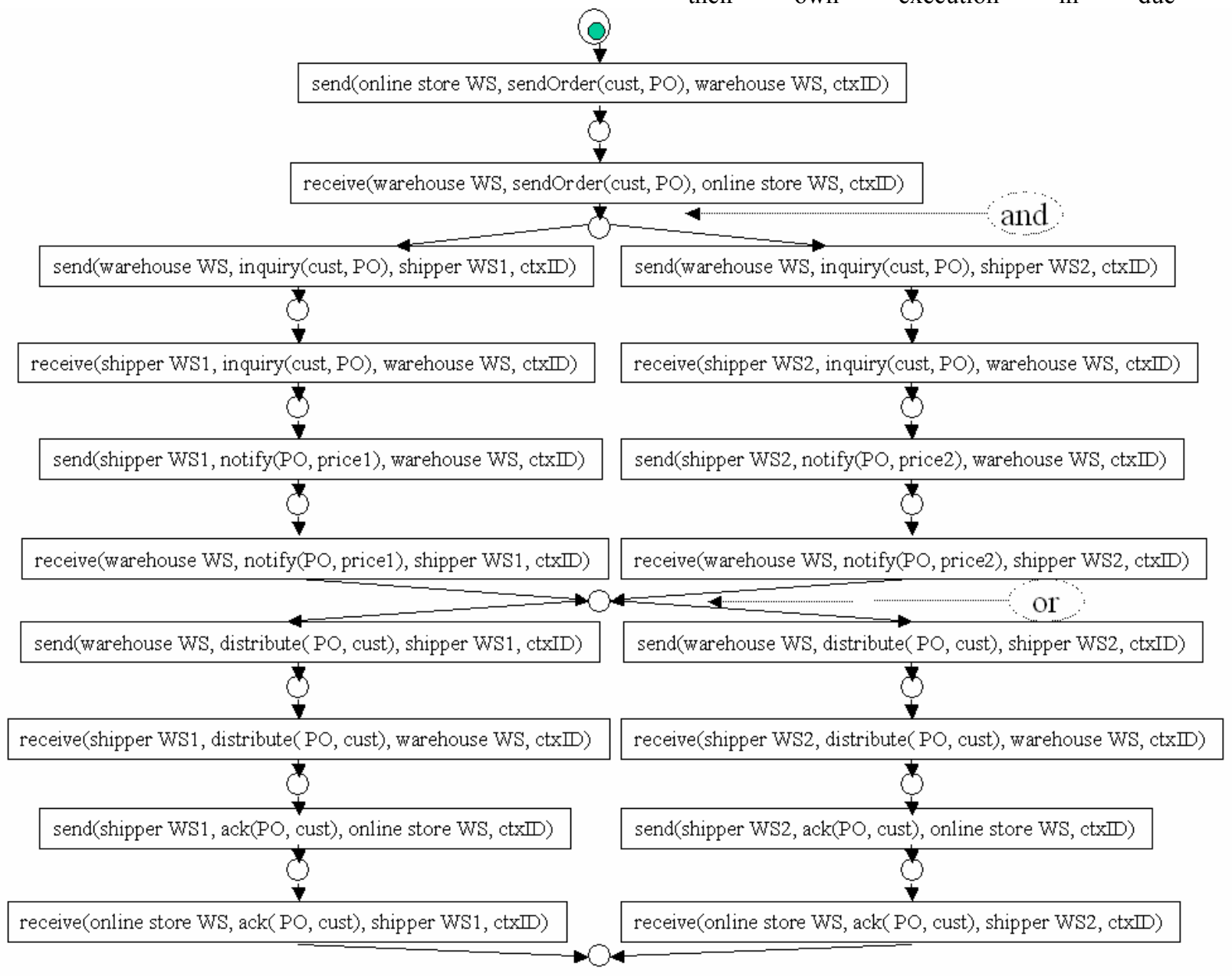

Fig. 2. Choreography specification of the online store example. 


\section{OUR FRAMEWORK}

\section{A. Choreography Specification}

The choreography of a complex service specifies the admissible message exchanges which may occur between all the cooperating Web Services at execution time.

It should be noticed that alternative service providers might be employed during different executions of the same choreographed service, because of availability problems or of different evolutions of the overall service. Therefore, it is convenient to generate the choreography specification at service execution time, instead of precompiling a large choreography which would include all the possible alternatives. For this reason, we have adopted an incremental approach to the specification of the choreography of a complex service. Our approach is based on the definition of local views on the choreography, held by the cooperating Web Services, and on the composition of such local views at service invocation time. The basic assumption is that each Web Service provider must publish the specification of its own interaction protocol (as suggested in Web Service conversation standards, e.g., WS-CDL [12] and WS-BPEL Abstract Processes), which might be manually defined by its own developer.

Let's suppose that the interaction protocol of each cooperating Web Service $W S_{i}$ is represented as an Abstract Process. Then, the local view of $W S_{i}$ on the choreography is determined by binding its Abstract Process to the endpoint Web Services interacting with $W S_{i}$. Moreover, the specification of the overall choreography may be achieved by composing the local views of all the cooperating Web Services.

We assume that, at choreographed service design time, the candidate Web Service providers to be invoked are selected by taking their Abstract Processes into account, and that the developer of the service solves any possible conflict due to heterogeneous interaction protocols (e.g., by adding mediators to the pool of cooperating Web Services [6]). Under these circumstances, the local views may be composed incrementally.

We have adopted a workflow language for the representation of the choreography because this is a possibly complex process; the only difference with standard workflows is the fact that the performed activities are conversational actions. For instance, Web Services may send messages sequentially, in parallel, or they may follow alternative message flow paths, which may split and join in the various phases of execution of the choreographed service. Specifically, we adopt a Reconfigurable Petri Net representation [3], which can be utilized to check whether the cooperating Web Services

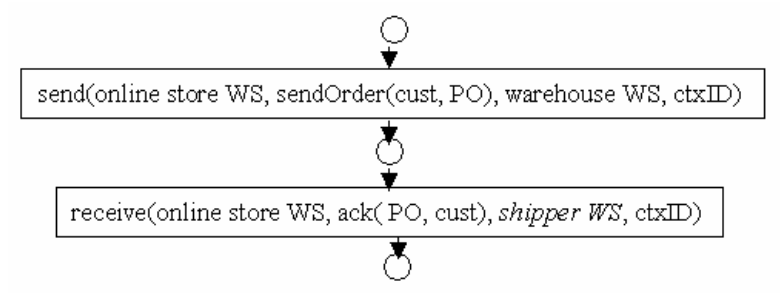

Fig. 3. Online store WS's local view on the choreography.

send messages as expected, and to represent the progress of the choreographed service by following the message flow paths corresponding to the sent/received messages. This type of Petri Net can be used to model the condition in which several choreographies are merged and thus the network topology changes during the service execution.

Figure 2 depicts the complete choreography specification of our sample scenario. Notice that the activities of the choreography have a $c t x I D$ parameter, which identifies the choreography instance to be considered by the Monitor. This is necessary because, at each time, the choreographed service might have multiple running instances; moreover, the Monitor might serve different choreographed services at the same time.

- Transitions represent the act of sending or receiving a message. A send transition specifies the sender, the object message (i.e., the message which has been sent), the recipient of the message and the choreography context identifier ctxID. The receive transitions are symmetric (they represent the conversational action performed by recipients) and specify recipient, object message, sender and choreography context ID.

- States represent the evolution of the choreographed service while messages are being sent or received.

Figures 3 and 4 depict the local views on the choreography held by the online store and the warehouse Web Services. In the figure, the roles not yet instantiated on concrete Web Service endpoints are shown in italics. For instance, initially, the online store WS does not know whether the shipper WS1 or WS2 will distribute the goods to the customer; thus, the shipper WS parameter is reported in italics.

\section{B. Initialization of the Choreography Monitoring Process}

Similar to the approach adopted in WS-Coordination, the first Web Service participating to a choreographed service invokes the Monitor, which generates a choreography coordination context and starts monitoring the messages between Web Services. Then, each invoked Web Service registers to the Monitor in order to join the pool of participants. As explained in Section III.D, when a 


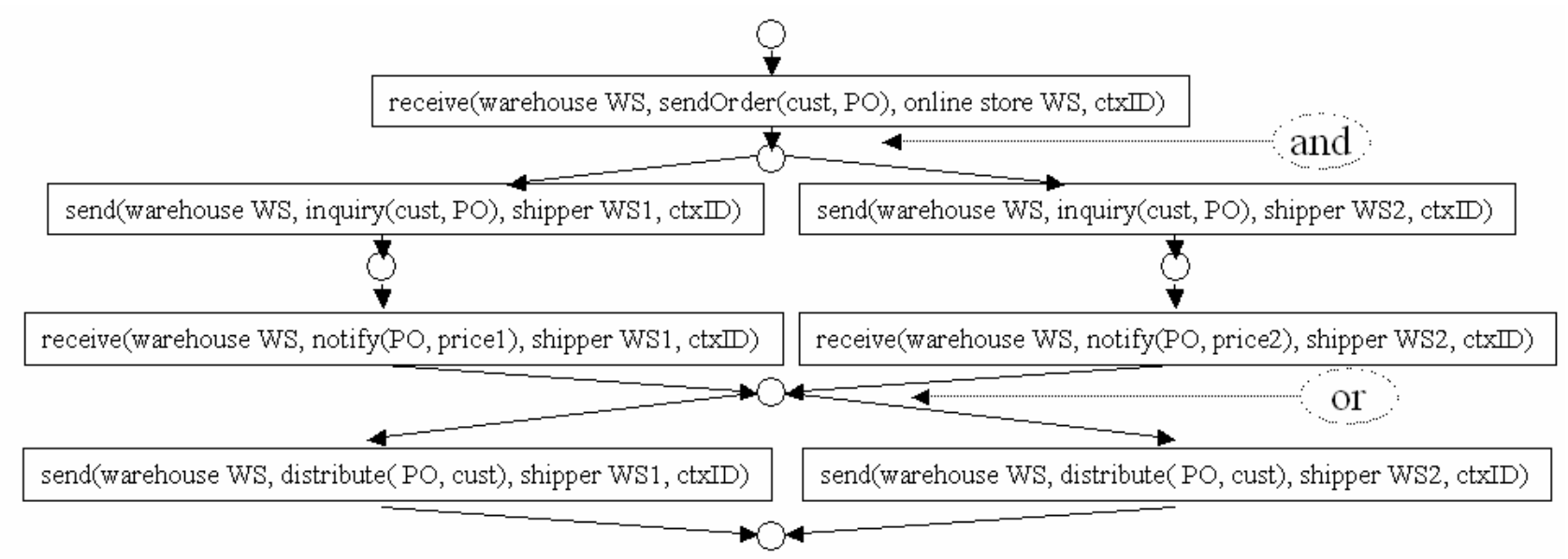

Fig. 4. Warehouse WS's local view on the choreography.

cooperating Web Service registers, it also provides the Monitor with its local view on the choreography. Thus, the Monitor starts with an empty choreography and incrementally extends it during the execution of the choreographed service.

As the choreography specification can be completed only after all the cooperating Web Services have registered, at each stage of execution, the Monitor can only detect interaction problems on the subset of the whole choreography built so far. However, this is sufficient for the early detection of several problems such as the identification of execution paths which cannot be successfully terminated because of an occurred fault.

\section{Choreography Monitoring}

During the execution of the choreographed service, the Monitor utilizes the partial service choreography to track the progress of the registered Web Services, and to identify possible execution problems which might obstacle their completion. Intuitively, while Web Services interact with one another, the Monitor follows their conversational behavior on the choreography net. The configuration of tokens in such a Petri Net describes the progress state of the choreographed service, from the conversational point of view. Specifically:

- The Monitor starts the choreography simulation from the initial state of the net.

- Each time a Web Service sends or receives a message, it notifies the Monitor about the event (see Section III.D). In turn, the Monitor performs a transition in the choreography net, moving tokens to represent the fact that a conversational action has been performed. As the choreography may include parallel paths, multiple nodes in the graph can be active at the same time. The active nodes represent the conversational state after several Web Services have sent or received messages.

If the cooperating Web Services successfully terminate the execution, the choreographed service completes and the Monitor reaches the final state of the choreography. Conversely, if a failure occurs, the Monitor analyzes the possible continuations of the interaction between the Web Services in order to identify which ones are affected by the problem. Thanks to the Petri Net representation, the Monitor can adopt existing dead path elimination algorithms to reason about the possible continuations of the interaction. For instance, given the current state of the choreography net and the information about the execution state of the Web Services (e.g., some is faulted), the Monitor can decide whether it is still possible to complete the choreographed service, and which Web Services should terminate their execution because they are involved in a dead path. Moreover, the Monitor may notify other affected Web Services about possible execution problems.

Let's consider our sample choreography and suppose that the shipper WS2 fails immediately after the warehouse WS has sent the two inquiry(cust, PO) messages. Then, the choreographed service may successfully continue if the warehouse WS invokes WS1 for the delivery. Nevertheless, the warehouse WS should be informed about the failure in order to avoid waiting for an answer from the shipper WS2, until the expiration of a time out. As another example, if the shipper WS1 fails after having received the distribute( $P O$, cust) message from the warehouse WS, it would be useful to inform the online store WS about the failure. In fact, the online store WS might immediately notify the end customer that the delivery of goods is going to be delayed. 


\section{Interaction between Cooperating Web Services and Monitor WS}

In order to be monitored and to be informed about execution problems concerning the choreographed service, the cooperating Web Services should offer a set of WSDL operations to be invoked by the Monitor WS; moreover, they should invoke some operations on the Monitor to inform it about the messages they send and/or receive. Specifically, the interaction between Monitor WS and cooperating Web Services is organized as follows:

- When an instance of the choreographed service is started, the first Web Service in execution sends the Monitor WS a startConversation(originatorWS) message.

- Upon receiving this message, a new instance of the Monitor WS is created. That instance creates a new choreography coordination context, registers the $\mathrm{Web}$ Service and returns a context ID by sending the Web Service a receive(ctxID) message. The Web Service also sends the Monitor its local view on the choreography (addChoreography message).

- When a registered Web Service invokes a Web Service provider, it adds the choreography coordination context ID (ctxID) to its own message. At the first invocation, the Web Service provider registers to the Monitor WS by specifying the ctxID (register(senderWS, ctxID)).

- Immediately after registration, each cooperating Web Service sends the Monitor WS a request to add its local view of the choreography to the partial choreography by sending a message of type addChoreography(senderWS, localView, ctxID).

- Each registered Web Service receives choreography coordination messages from the Monitor WS. Moreover, the Web Service can send messages to inform the Monitor about changes in the execution state. For instance, the Web Service might notify the Monitor about an occurred failure or about its successful termination.

- The Monitor WS can proactively send getStatus messages to the cooperating Web Services, which respond with status messages describing their own execution state. The getStatus message can be seen as a sort of ping: if the invoked Web Service does not respond in due time, this means that it is unavailable. ${ }^{1}$

\footnotetext{
1 A missing reply might also mean that the network connection between Web Service and Monitor is down. In order to take this issue into account, we will extend the interaction protocol to support information redundancy.
}

We assume that the registered Web Services may send the Monitor various types of messages in order to inform it about their activities. To this purpose, the Monitor WS offers the following WSDL operations:

- send(senderWS, msg, destWS, ctxID) informs the Monitor that the sender WS has sent message $m s g$ to the destination WS, within the instance of the choreography identified by $c t x I D$.

- receive(destWS, $m s g$, senderWS, ctxID) informs the Monitor that message msg has been received by the recipient Web Service.

- startConversation(originatorWS): the Web Service starting the choreographed service notifies the Monitor that the process has started and has to be monitored.

- addChoreography(senderWS, localView, ctxID): the senderWS Web Service sends the Monitor its own local view of the choreography, by specifying the choreography instance to be considered (ctxID).

- register(senderWS, ctxID) notifies the Monitor that senderWS joins the service.

- status(ctxID, currentStatus): upon receiving a getStatus message from the Monitor, the Web Service sends its current execution status.

In order to test our framework, we have developed a WS-monitoring Java class, which implements the WSDL operations to be offered by a cooperating Web Service, and can be exploited by a Web Service supplier to interact with the Monitor WS without modifying its own business logic; e.g., the class may be used within message handlers associated with each cooperating Web Service. Moreover, we have developed a Monitor WS which runs a choreography net in order to track the progress of a choreographed service. The Monitor is based on the JBPM workflow management tool and the choreography net is implemented as a JBPM process.

\section{DISCUSSION}

We have described a monitoring framework supporting the early detection of faults in choreographed services, i.e., complex services characterized by the cooperation of multiple service providers, none of which has complete control on the overall business logic. Our framework is based on the introduction of a Monitor Web Service, which maintains a snapshot of the multi-party conversations carried out by the cooperating Web Services. When a failure occurs in an instance of the choreographed service, the Monitor analyzes the choreography specification to decide whether it is still possible to continue the service. Moreover, the Monitor notifies the service providers which cannot continue their execution, in order to let them react as needed. 
Our monitoring framework builds on the WSCoordination standard [2] for the management of the coordination context between a set of cooperating Web Services. However, our Monitor WS offers additional features with respect to a standard Coordinator; e.g., it composes the local views on the choreography held by the Web Services into a unified one; moreover, it analyzes the evolution of the choreography in order to assess its progress state and notify the cooperating Web Services about execution problems.

Indeed, there are similarities between our approach and the one followed in WSBusinessActivity, and some of the messages exchanged between cooperating Web Services and Monitor WS are the same as those defined in that standard. However:

- We introduce new message types in order to inform the Monitor about which choreography paths are traversed during the execution of the overall service.

- The Monitor utilizes the choreography specification to evaluate the possibility of success of the overall service, depending on the execution state of the cooperating Web Services and on which portion of the choreography has been completed. The reason is that, while the failure of a business activity determines the failure of the whole scope to which the activity belongs, the occurrence of a failure during the execution of a choreography does not necessarily cause the failure of the whole service. In fact, alternative courses of actions may exist, which enable the service to successfully complete.

Our framework could be extended to provide additional monitoring features. For instance, it would be interesting to deal with delays in service execution in order to address some typical Quality of Service concerns. To this purpose, the Monitor WS should be extended with a temporal reasoning module which estimates the time needed to complete a choreographed service. If timing information were added to the specification of a choreography (e.g., time outs and expected time to elapse between messages), the Monitor might compute the expected service execution time and check the progress of the service. This would enable the Monitor to evaluate whether it is still possible to complete the service on time, or if any admissible conversation paths have to be excluded because they would violate the overall time constraints.

Adding a Monitor to a choreographed service is interesting only when the interaction between the cooperating Web Services is complex and simply restarting the whole process is costly. In fact, monitoring imposes an obvious overhead due to an increase in messages exchanged between the Web Services and the Monitor. Moreover, monitoring the progress of a choreographed service raises serious applicability issues; on the one hand, the introduction of a centralized observer controlling the overall message flow is knowledge intensive, unless the choreography specification can be automatically generated out of the local views of the cooperating Web Services. On the other hand, various languages are being proposed to specify the interaction protocol of a Web Service (e.g., BPEL Abstract Processes [7] and automata-based approaches such as the Abstract State Machines [1]) and the choreography of a set of cooperating Web Services (e.g., WSCDL [12] and Petri Nets [11]). However, an agreement on representation formalisms has not been reached so far; e.g., see [4]. Therefore, the choreographed service might need to abstract from heterogeneous representations of the interaction protocols of the individual service providers.

\section{ACKNOWLEDGMENT}

The work described in this paper has been partially funded by the EU Project WS-DIAMOND (grant IST516933) and the MIUR Project QuaDRAnTIS.

\section{REFERENCES}

[1] M. Altenhofen, E. Börger, and J. Lemcke. An abstract model for process mediation. In Proc. 7th Int. Conf. on Formal Engineering Methods, pp. 81-95, Manchester, UK, 2005.

[2] Arjuna. Introducing WS-Coordination - an Arjuna Technologies report. http://www.arjuna.com/library/reports/2003-04-WSCoordination-intro.pdf, 2003.

[3] E. Badouel and J. Oliver. Reconfigurable Nets, a class of high level Petri Nets supporting dynamic changes. Technical Report PI1163, Inria, 1998.

[4] A. Barros, M. Dumas, and P. Oaks. A critical overview of the Web Services Choreography Description Language (WS-CDL). BPTrends, March, 2005.

[5] G. Chafle, S. Chandra, V. Mann, and M.G. Nanda. Decentralized orchestration of composite Web Services. In Proc. of 13th Int. World Wide Web Conferenc, pp. 134-143, New York, 2004.

[6] DERI International. Web ServiceModelling eXecution environment. http://www.wsmx.org/, 2006.

[7] OASIS. OASIS Web Services Business Process Execution Language.

http://www.oasisopen.org/committees/documents.php?wg abbrev=wsbpel, 2005

[8] OASIS. OASIS Web Services Business Activity Framework (WSBusinessActivity). http://schemas.xmlsoap.org/ws/2004/10/wsba/, 2006.

[9] M.P. Papazoglou and D. Georgakopoulos. Service-Oriented Computing. Communications of the ACM, 46(10), 2003.

[10] C. Peltz. Web Services orchestration and choreography. Innovative Technology for Computing Professionals, 36(10):46-52, 2003.

[11] W. van der Aalst, M. Dumas, C. Ouyang, A. Rozinat, and H.M.W. Verbeek. Choreography conformance checking: an approach based on BPEL and Petri Nets. In ePrints Archive, Adelaide, South Australia, 2006.

[12] W3C. Web Services Choreography Description Language version 1.0. http://www.w3.org/TR/ws-cdl-10/, 2005. 